\title{
CONTRE-EXEMPLES AU PRINCIPE DE HASSE POUR CERTAINS TORES COFLASQUES
}

\author{
par \\ R. de la Bretèche \& T.D. Browning
}

\begin{abstract}
Résumé. - Nous étudions le comportement asymptotique du nombre de variétés dans une certaine classe ne satisfaisant pas le principe de Hasse. Cette étude repose sur des résultats récemment obtenus par Colliot-Thélène [3].
\end{abstract}

\section{Introduction}

Nous nous intéressons à la fréquence de contre-exemples au principe de Hasse dans une famille de variétés algébriques définies sur $\mathbb{Q}$. Les courbes de degré 3 dans $\mathbb{P}_{\mathbb{Q}}^{2}$ sont l'objet du travail de Bhargava [1. Le cas des surfaces de Châtelet a été récemment étudié par La Bretèche et Browning [2].

Le but de cet article est de faire de même pour les variétés affines $Y \subset \mathbb{A}_{\mathbb{Q}}^{6}$, définies par

$$
\left(x^{2}-a y^{2}\right)\left(z^{2}-b t^{2}\right)\left(u^{2}-a b w^{2}\right)=c,
$$

avec $a, b, c \in \mathbb{Q}^{*}$. L'arithmétique de $Y$ a été étudiée par Colliot-Thélène [3, §5], qui a notamment montré que le choix de coefficients $(a, b, c)=(13,17,5)$ donne un contreexemple au principe de Hasse. Notre investigation quantitative est fondée sur son travail.

La variété $Y$ est un espace principal homogène du tore coflasque

$$
\left(x^{2}-a y^{2}\right)\left(z^{2}-b t^{2}\right)\left(u^{2}-a b w^{2}\right)=1 .
$$

D'après un résultat de Sansuc [5, Cor. 8.7], l'obstruction Brauer-Manin est la seule obstruction au principe de Hasse. Soit $Y^{c}$ une $\mathbb{Q}$-compactification lisse de $Y$ et $\overline{Y^{c}}=Y^{c} \times_{\mathbb{Q}} \overline{\mathbb{Q}}$. Une caractéristique intéressante de $Y$ est le fait qu'il existe un générateur universel explicite pour le groupe de $\operatorname{Brauer} \operatorname{Br}\left(Y^{c}\right) / \operatorname{Br}(\mathbb{Q})=H^{1}\left(\operatorname{Gal}(\overline{\mathbb{Q}} / \mathbb{Q})\right.$, $\left.\operatorname{Pic}\left(\overline{Y^{c}}\right)\right)$.

En fait, suite à $\left[3\right.$, Thm. 4.1], si $a, b, a b \in \mathbb{Q}^{*} \backslash \mathbb{Q}^{* 2}$ on a $\operatorname{Br}\left(Y^{c}\right) / \operatorname{Br}(\mathbb{Q})=\mathbb{Z} / 2 \mathbb{Z}$ avec l'algébre de quarternions $\left(x^{2}-a y^{2}, b\right) \in \operatorname{Br}(\mathbb{Q}(Y))$ comme générateur, tandis que, si l'un des $a, b, a b$ est dans $\mathbb{Q}^{* 2}, Y$ est $\mathbb{Q}$-rationnelle, et donc $\operatorname{Br}\left(Y^{c}\right) / \operatorname{Br}(\mathbb{Q})=0$. Nous utilisons cette 
description explicite pour déterminer la fréquence à laquelle il existe des contre-exemples au principe de Hasse pour les variétés (1.1).

Nous paramétrons les variétés $Y$ par l'ensemble

$$
S=\left\{(a, b, c) \in(\mathbb{Z} \backslash\{0\})^{3}: a, b, c \text { sans facteur carré et } c>0\right\} .
$$

Il est évident que toute $\mathbb{Q}$-variété (1.1) est $\mathbb{Q}$-isomorphe à la variété définie par la même équation avec $(a, b, c) \in S$. Notre intérêt principal est de déterminer la répartition des éléments de $S$ tels que $Y(\mathbb{Q})$ est vide (ou non-vide). À la lumière de [3, Prop. 5.1(a)], pour toute place $v$ de $\mathbb{Q}$ et chaque $(a, b, c) \in S$, on a $Y\left(\mathbb{Q}_{v}\right) \neq \emptyset$. Il n'y a donc jamais d'obstruction locale pour l'existence de $\mathbb{Q}$-points.

Soit $S(P)=\{(a, b, c) \in S: \max \{|a|,|b|, c\} \leqslant P\}$, pour $P \geqslant 1$. Nous estimons asymptotiquement, lorsque $P$ tend vers l'infini, le cardinal

$$
N_{\mathrm{Br}}(P)=\#\{(a, b, c) \in S(P): Y(\mathbb{Q})=\emptyset\} .
$$

Notre résultat principal est le suivant.

Théorème 1.1. - Lorsque $P \geqslant 2$, on a

$$
N_{\mathrm{Br}}(P)=\frac{\tau_{1} P^{3}}{\log P}-\frac{\tau_{2} P^{3}}{(\log P)^{\frac{3}{2}}}+O\left(\frac{P^{3}}{(\log P)^{2}}\right)
$$

où

$$
\begin{aligned}
& \tau_{1}=\frac{45}{\pi^{5}} \prod_{p>2}\left(1+\frac{1}{2 p(p+1)}\right)+\frac{15}{\pi^{5}} \prod_{p>2}\left(1+\frac{(-1)^{\frac{p-1}{2}}}{2 p(p+1)}\right), \\
& \tau_{2}=\frac{153}{16 \pi^{\frac{7}{2}}} \prod_{p} \frac{\left(1-\frac{1}{p}\right)^{\frac{1}{2}}}{\left(1+\frac{1}{p}\right)}\left(1+\frac{3}{2 p}+\frac{1}{p^{2}}\right) .
\end{aligned}
$$

La différence $N_{\text {glob }}(P)=\# S(P)-N_{\mathrm{Br}}(P)$ est le nombre de variétés $Y$ paramétrées par $S(P)$ pour lesquelles $Y(\mathbb{Q}) \neq \emptyset$. Le cardinal $\# S(P)$ étant facile à estimer, nous obtenons le résultat suivant.

Corollaire 1.2. - Lorsque $P \geqslant 2$, on a

$$
\begin{aligned}
N_{\text {glob }}(P) & =\# S(P)+O\left(\frac{P^{3}}{\log P}\right) \\
& =\frac{864}{\pi^{6}} P^{3}+O\left(\frac{P^{3}}{\log P}\right) .
\end{aligned}
$$

En particulier, on a une proportion asymptotique de $100 \%$ des variétés Y qui ont des $\mathbb{Q}$ points.

Remerciements. - Pendant l'élaboration de cet article, le premier auteur a été soutenu par un IUF junior et le projet $A N R$ (PEPR), tandis que le second auteur a été soutenu par la bourse ERC $30645 \%$. 


\section{L'obstruction Brauer-Manin}

Nous rappelons quelques points clés du travail de Colliot-Thélène [3], sur les variétés $Y$ définies en (1.1), lorsque $(a, b, c)$ appartient à l'ensemble $S$ défini en (1.2).

Selon [3, Prop. 5.1(c)], on a $Y(\mathbb{Q}) \neq \emptyset$ s'il existe un nombre premier $p$ tel qu'aucun des $a, b, a b$ ne soit pas un carré dans $\mathbb{Q}_{p}^{*}$. Supposons que pour chaque premier $p$ l'un au moins des $a, b$ ou $a b$ est un carré dans $\mathbb{Q}_{p}^{*}$, alors il découle de $[3$, Prop. $5.1(\mathrm{~d})]$ que $Y(\mathbb{Q})=\emptyset$ si, et seulement si,

$$
\sum_{\substack{p \\ a \notin \mathbb{Q}_{p}^{* 2}}}[c, b]_{p} \equiv 1(\bmod 2) .
$$

Ici $[\cdot, \cdot]_{p}: \mathbb{Q}_{p}^{*} \times \mathbb{Q}_{p}^{*} \rightarrow \mathbb{Z} / 2 \mathbb{Z}$ est défini par $(\cdot, \cdot)_{p}=(-1)^{[\cdot, \cdot]_{p}}$, où $(\cdot, \cdot)_{p}$ est le symbole de Hilbert.

Lorsque $(a, b, c) \in \mathbb{Z}^{3}$, nous considérons

$$
f(a, b)= \begin{cases}1, & \text { si } a, b \text { ou } a b \text { est dans } \mathbb{Q}_{p}^{* 2} \text { pour tout } p \\ 0, & \text { sinon }\end{cases}
$$

et

$$
h(a, b, c)=\prod_{\substack{p \\ a \notin \mathbb{Q}_{p}^{* 2}}}(c, b)_{p} .
$$

Notre problème est donc d'évaluer, lorsque $P$ tend vers l'infini, la quantité

$$
\begin{aligned}
N_{\mathrm{Br}}(P) & =\sum_{(a, b, c) \in S(P)} \frac{f(a, b)}{2}(1-h(a, b, c)) \\
& =\frac{1}{2}\left(N_{1}(P)-N_{2}(P)\right),
\end{aligned}
$$

où les définitions de $N_{1}(P)$ et $N_{2}(P)$ sont évidentes. Notre analyse de $N_{1}(P)$ et $N_{2}(P)$ est inspirée du travail de Friedlander et Iwaniec [4].

Nous commençons avec l'observation

$$
N_{1}(P)=\sum_{(a, b, c) \in S(P)} f(a, b)=\left(\sum_{\substack{(a, b) \in \mathbb{Z}^{2} \\|a|,|b| \leqslant P}} \mu^{2}(a) \mu^{2}(b) f(a, b)\right)\left(\sum_{1 \leqslant c \leqslant P} \mu^{2}(c)\right),
$$

où $\mu$ est la fonction de Möbius. Nous étendons la définition de la fonction $\mu$ de telle sorte que $\mu(0)=0$. Le deuxième facteur est facile à estimer. Il vient

$$
N_{1}(P)=\frac{6 P}{\pi^{2}} \sum_{\substack{(a, b) \in \mathbb{Z}^{2} \\|a|,|b| \leqslant P}} \mu^{2}(a) \mu^{2}(b) f(a, b)+O\left(P^{\frac{5}{2}}\right) .
$$

Il est clair que l'un au moins des $a, b$ ou $a b$ est un carré dans $\mathbb{Q}_{p}^{*}$ pour chaque premier $p \nmid 2 a b$. Quand $p=2$ et $a b$ est impair, la condition relative à $p=2$ contenue dans $f(a, b)$ 
est que l'un au moins des $a, b$ or $a b$ est congru à 1 modulo 8. Rappelant que $a, b$ sont des entiers sans facteur carré, nous avons alors l'égalité

$$
\begin{aligned}
f(a, b)= & f_{2}(a, b) \prod_{\substack{p \mid a \\
p \nmid 2 b}} \frac{1}{2}\left(1+\left(\frac{b}{p}\right)\right) \prod_{\substack{p \mid b \\
p \nmid 2 a}} \frac{1}{2}\left(1+\left(\frac{a}{p}\right)\right) \\
& \times \prod_{\substack{p \mid \operatorname{gcd}(a, b) \\
p \neq 2}} \frac{1}{2}\left(1+\left(\frac{a b /(a, b)^{2}}{p}\right)\right),
\end{aligned}
$$

avec

$$
f_{2}(a, b)= \begin{cases}1, & \text { si } 2 \nmid a b \text { et } 1 \in\{a, b, a b\}(\bmod 8), \\ 1, & \text { si } 2 \mid a \text { et } b \equiv 1(\bmod 8), \\ 1, & \text { si } 2 \mid b \text { et } a \equiv 1(\bmod 8), \\ 1, & \text { si } 2 \mid(a, b), a b \equiv 4(\bmod 32), \\ 0, & \text { sinon. }\end{cases}
$$

Avec $\vartheta$ défini sur les impairs par $\vartheta(k)=1$ si $k \equiv 3(\bmod 4)$ et $\vartheta(k)=0$ si $k \equiv 1(\bmod 4)$, la loi de réciprocité quadratique s'énonce, lorsque $k$ et $\ell$ sont des nombres entiers impairs premiers entre eux, sous la forme

$$
\left(\frac{k}{\ell}\right)\left(\frac{\ell}{k}\right)=(-1)^{\vartheta(k) \vartheta(\ell)} .
$$

Nous terminons cette section par quelques mots sur les symboles de Hilbert (cf. [6, §3]). Soit $p$ un nombre premier et $x, y \in \mathbb{Q}_{p}^{*}$. Supposant que $x=p^{\xi} u$ et $y=p^{\eta} v$, où $u, v$ sont des $p$-unités, alors pour tout $p>2$ nous avons

$$
(x, y)_{p}=(-1)^{\xi \eta \vartheta(p)}\left(\frac{u}{p}\right)^{\eta}\left(\frac{v}{p}\right)^{\xi}
$$

tandis que lorsque $p=2$, nous avons

$$
(x, y)_{2}=(-1)^{\vartheta(u) \vartheta(v)+\frac{\xi\left(v^{2}-1\right)}{8}+\frac{\eta\left(u^{2}-1\right)}{8} .}
$$

\section{Lemmes techniques}

Lorsque $\nu \in \mathbb{Z}_{\geqslant 0}$, nous considérons

$$
\varphi_{\nu}(r)=\prod_{p \mid r}\left(1+\frac{\nu}{2 p}\right)^{-1} .
$$

Nous aurons besoin de [4, Cor. 2], concernant

$$
C_{\nu}(x ; a, d, r, q, \chi)=\sum_{\substack{n \leqslant x \\(n, d)=1 \\ n \equiv a(\bmod r)}} \frac{\mu^{2}(n) \chi(n)}{2^{\omega(n)}} \varphi_{\nu}(n),
$$


où $\chi$ est un caractère modulo $q$ et $\omega(n)$ désigne le nombre de facteurs premiers distincts de $n$. Posons

$$
c_{\nu}(r)=c_{\nu}(1) \prod_{p \mid r}\left(1+\frac{\varphi_{\nu}(p)}{2 p}\right)^{-1}
$$

avec

$$
c_{\nu}(1)=\frac{1}{\sqrt{\pi}} \prod_{p}\left(1+\frac{\varphi_{\nu}(p)}{2 p}\right)\left(1-\frac{1}{p}\right)^{\frac{1}{2}} .
$$

Notons aussi $\delta$ la fonction caractéristique des caractères principaux.

Lemme 3.1. - Soient $A>0$ fixé et $\nu \in\{0,1,2\}$. Lorsque $a, d, r, q$ sont des entiers satisfaisant $(a, r)=(d, r q)=(r, q)=1, x \geqslant 2$ et $\chi$ est un caractère modulo $q$, on a

$$
C_{\nu}(x ; a, d, r, q, \chi)=\delta(\chi) \frac{c_{\nu}(d r q)}{\varphi(r)} \frac{x}{\sqrt{\log x}}\left\{1+O\left(\frac{(\log 3 d r q)^{\frac{3}{2}}}{\log x}\right)\right\}+O\left(\frac{\tau(d) r q x}{(\log x)^{A}}\right) .
$$

Démonstration. - Dans [4, Cor. 2], ce résultat est démontré pour $\nu=0$, la condition supplémentaire $(a, q)=1$ étant inutile. Les cas $\nu=1,2$ se démontrent de la même manière.

Soient $\mathbf{x}=\left(x_{1}, x_{2}\right), \mathbf{a}=\left(a_{1}, a_{2}\right), \mathbf{d}=\left(d_{1}, d_{2}\right), \mathbf{r}=\left(r_{1}, r_{2}\right)$ et $\mathbf{q}=\left(q_{1}, q_{2}\right)$. Du Lemme 3.1, nous déduisons l'estimation de la somme

$$
Q\left(\mathbf{x} ; \mathbf{a}, \mathbf{d}, r, \mathbf{q}, \chi_{1}, \chi_{2}\right)=\sum_{\substack{\left(n_{1}, n_{2}\right) \in \mathbb{N}^{2} \\ n_{i} \leqslant x_{i} \\\left(n_{i}, d_{i}\right)=1,\left(n_{1}, n_{2}\right)=1 \\ n_{i} \equiv a_{i}(\bmod r)}} \mu^{2}\left(n_{1} n_{2}\right) \frac{\chi_{1}\left(n_{1}\right) \chi_{2}\left(n_{2}\right)}{2^{\omega\left(n_{1} n_{2}\right)}},
$$

où les $\chi_{i}$ sont des caractères modulo $q_{i}$.

Corollaire 3.2. - Soit $A>0$ fixé. Lorsque $a_{1}, a_{2}, d_{1}, d_{2}, r, q_{1}, q_{2}$ sont des entiers satisfaisant $\left(a_{i}, r\right)=\left(d_{i}, q_{i}\right)=\left(r, d_{1} d_{2} q_{1} q_{2}\right)=1, x_{i} \geqslant 2$ et $\chi_{i}$ sont des caractères modulo $q_{i}$, on $a$

$$
\begin{aligned}
Q\left(\mathbf{x} ; \mathbf{a}, \mathbf{d}, r, \mathbf{q}, \chi_{1}, \chi_{2}\right)= & \delta\left(\chi_{1}\right) \delta\left(\chi_{2}\right) \frac{c(\mathbf{d}, r)}{\varphi(r)^{2}} \frac{x_{1} x_{2}}{\sqrt{\log x_{1} \log x_{2}}}\left\{1+O\left(\frac{\left(\log 3 d_{1} d_{2} r q_{1} q_{2}\right)^{\frac{3}{2}}}{\log \min \left\{x_{1}, x_{2}\right\}}\right)\right\} \\
& +O\left(\frac{\tau\left(d_{1} d_{2}\right) r q_{1} q_{2} x_{1} x_{2}}{\left(\log \min \left\{x_{1}, x_{2}\right\}\right)^{A}}\right)
\end{aligned}
$$

avec

$$
c(\mathbf{d}, r)=\frac{6}{\pi^{3}} \frac{\varphi_{1}\left(d_{1} r\right) \varphi_{1}\left(d_{2} r\right)}{\varphi_{1}\left(d_{1} d_{2} r\right)^{2}} \varphi_{2}\left(d_{1} d_{2} r\right) .
$$

Démonstration. - Notons $Q$ la quantité à estimer. Une interversion de Möbius fournit

$$
Q=\sum_{\substack{n \in \mathbb{N} \\\left(n, d_{1} d_{2} r q_{1} q_{2}\right)=1}} \frac{\mu(n) \chi_{1}(n) \chi_{2}(n)}{4^{\omega(n)}} C_{0}\left(\frac{x_{1}}{n} ; n^{-1} a_{1}, d_{1} n, r, q_{1}, \chi_{1}\right) C_{0}\left(\frac{x_{2}}{n} ; n^{-1} a_{2}, d_{2} n, r, q_{2}, \chi_{2}\right) .
$$


Nous pouvons restreindre la sommation aux entiers $n \leqslant T$ où $T=\left(\log \min \left\{x_{1}, x_{2}\right\}\right)^{\frac{A}{2}}$, la contribution complémentaire étant majorée par $O\left(x_{1} x_{2} / T\right)$. Nous appliquons ensuite le Lemme 3.1 avec $\nu=0$. Il vient

$$
\begin{aligned}
Q= & \delta\left(\chi_{1}\right) \delta\left(\chi_{2}\right) \sum_{\substack{n \leqslant T \\
\left(n, d_{1} d_{2} r\right)=1}} \frac{\mu(n) c_{0}\left(d_{1} n r\right) c_{0}\left(d_{2} n r\right) x_{1} x_{2}}{4^{\omega(n)} n^{2} \varphi(q)^{2} \sqrt{\log x_{1} \log x_{2}}}\left\{1+O\left(\frac{\left(\log 3 d_{1} d_{2} r q_{1} q_{2}\right)^{\frac{3}{2}}}{\log \left(\min \left\{x_{1}, x_{2}\right\}\right)}\right)\right\} \\
& +O\left(\frac{\tau\left(d_{1} d_{2}\right) r q_{1} q_{2} x_{1} x_{2} T}{\left(\log \left(\min \left\{x_{1}, x_{2}\right\}\right)\right)^{A}}+\frac{x_{1} x_{2}}{T}\right) \\
= & \delta\left(\chi_{1}\right) \delta\left(\chi_{2}\right) \frac{c(\mathbf{d}, r) x_{1} x_{2}}{\varphi(q)^{2} \sqrt{\log x_{1} \log x_{2}}}\left\{1+O\left(\frac{\left(\log 3 d_{1} d_{2} r q_{1} q_{2}\right)^{\frac{3}{2}}}{\log \min \left\{x_{1}, x_{2}\right\}}\right)\right\} \\
& +O\left(\frac{\tau\left(d_{1} d_{2}\right) r q_{1} q_{2} x_{1} x_{2}}{\left(\log \min \left\{x_{1}, x_{2}\right\}\right)^{\frac{A}{2}}}\right) .
\end{aligned}
$$

Ici nous avons

$$
\begin{aligned}
c(\mathbf{d}, r) & =c_{0}\left(d_{1} r\right) c_{0}\left(d_{2} r\right) \sum_{\substack{n \in \mathbb{N} \\
\left(n, d_{1} d_{2} r\right)=1}} \frac{\mu(n) \varphi_{1}(n)^{2}}{4^{\omega(n)} n^{2}} \\
& =\frac{1}{\pi} \varphi_{1}\left(d_{1} r\right) \varphi_{1}\left(d_{2} r\right) \prod_{p \mid d_{1} d_{2} r}\left(1-\frac{1}{(2 p+1)^{2}}\right)^{-1} \prod_{p}\left(1-\frac{1}{p^{2}}\right) \\
& =\frac{6}{\pi^{3}} \varphi_{1}\left(d_{1} r\right) \varphi_{1}\left(d_{2} r\right) \frac{\varphi_{2}\left(d_{1} d_{2} r\right)}{\varphi_{1}\left(d_{1} d_{2} r\right)^{2}}
\end{aligned}
$$

ce qui fournit donc le résultat quitte à modifier la valeur du paramètre $A$.

Lorsque $q_{1} q_{2}$ est grand, le Corollaire 3.2 est inutilisable. Nous aurons besoin ainsi du résultat suivant [4, Lemme 2].

Lemme 3.3. - Soient $\left\{\alpha_{m}\right\}_{m \in \mathbb{N}},\left\{\beta_{n}\right\}_{n \in \mathbb{N}}$ des suites de nombres complexes telles que $\left|\alpha_{m}\right|,\left|\beta_{n}\right| \leqslant 1$, dont le support est inclus dans les nombres impairs. Lorsque $M, N \geqslant 1$, on $a$

$$
\sum_{m \leqslant M} \sum_{n \leqslant N} \alpha_{m} \beta_{n}\left(\frac{n}{m}\right) \ll\left(M N^{\frac{5}{6}}+N M^{\frac{5}{6}}\right)(\log 3 M N)^{\frac{7}{6}}
$$

\section{4. Étude de $N_{1}(P)$}

Pour estimer $N_{1}(P)$ à partir de (2.3), nous considérons

$$
T(P)=\sum_{\substack{(a, b) \in \mathbb{Z}^{2} \\|a|,|b| \leqslant P}} \mu^{2}(a) \mu^{2}(b) f(a, b) .
$$


Lorsque $\varepsilon=\left(\varepsilon_{1}, \varepsilon_{2}\right) \in\{ \pm 1\}^{2}$ et $\alpha, \beta \in\{0,1\}$, nous notons $T(P ; \alpha, \beta, \varepsilon)$ la contribution dans $T(P)$ des couples $(a, b)$ tels que $2^{\alpha} \| a$ et $2^{\beta} \| b, \varepsilon_{1} a>0, \varepsilon_{2} b>0$. Les couples $\left(a 2^{-v_{2}(a)}, b 2^{-v_{2}(b)}\right)$ appartiennent à un ensemble $E_{\alpha, \beta}$ modulo 8 , avec

$$
\begin{aligned}
& E_{0,0}=\{(1, \pm 1),(1, \pm 3),(-1, \pm 1),( \pm 3,1),( \pm 3, \pm 3)\} \\
& E_{1,0}=\{( \pm 1,1),( \pm 3,1)\} \\
& E_{0,1}=\{(1, \pm 1),(1, \pm 3)\} \\
& E_{1,1}=\{( \pm 1, \pm 1),( \pm 3, \pm 3)\}
\end{aligned}
$$

Lorsque $\varepsilon \in\{ \pm 1\}^{2}$ et $\left(a 2^{-\alpha}, b 2^{-\beta}\right) \equiv\left(a_{0}, b_{0}\right)(\bmod 8)$ avec $\left(a_{0}, b_{0}\right) \in E_{\alpha, \beta}, \varepsilon_{1} a>0$, et $\varepsilon_{2} b>0$, la formule (2.4) s'écrit aussi

$$
\mu^{2}(a) \mu^{2}(b) f(a, b)=\sum_{\substack{\left(k, k^{\prime}, \ell, \ell^{\prime}, m, m^{\prime}\right) \in \mathbb{N}^{6} \\ a=\varepsilon_{1} 2^{\alpha} k k^{\prime} m m^{\prime} \\ b=\varepsilon_{2} 2^{\beta} \ell \ell^{\prime} m m^{\prime}}} \frac{\mu^{2}\left(2 k k^{\prime} m m^{\prime} \ell \ell^{\prime}\right)}{2^{\omega\left(k k^{\prime} \ell \ell^{\prime} m m^{\prime}\right)}}\left(\frac{b}{k}\right)\left(\frac{a}{\ell}\right)\left(\frac{a b /(a, b)^{2}}{m}\right) .
$$

Il vient

$$
\begin{aligned}
T(P ; \alpha, \beta, \boldsymbol{\varepsilon})= & \sum_{\left(a_{0}, b_{0}\right) \in E_{\alpha, \beta}} \sum_{\begin{array}{c}
\left(k, k^{\prime}, \ell, \ell^{\prime}, m, m^{\prime}\right) \in \mathbb{N}^{6} \\
2^{\alpha} k k^{\prime} m m^{\prime}, 2^{\beta} \ell \ell^{\prime} m m^{\prime} \leqslant P \\
\left(\varepsilon_{1} k k^{\prime} m m^{\prime}, \varepsilon_{2} \ell \ell^{\prime} m m^{\prime}\right) \equiv\left(a_{0}, b_{0}\right)(\bmod 8)
\end{array}} \frac{\mu^{2}\left(2 k k^{\prime} m m^{\prime} \ell \ell^{\prime}\right)}{2^{\omega\left(k k^{\prime} \ell \ell^{\prime} m m^{\prime}\right)}} \\
& \times\left(\frac{\varepsilon_{2} 2^{\beta} \ell \ell^{\prime} m m^{\prime}}{k}\right)\left(\frac{\varepsilon_{1} 2^{\alpha} k k^{\prime} m m^{\prime}}{\ell}\right)\left(\frac{\varepsilon_{1} \varepsilon_{2} 2^{\alpha+\beta} k k^{\prime} \ell \ell^{\prime}}{m}\right) .
\end{aligned}
$$

La loi de réciprocité quadratique (2.5) et la multiplicativité des caractères fournissent

$$
\left(\frac{\varepsilon_{2} 2^{\beta} \ell \ell^{\prime} m m^{\prime}}{k}\right)\left(\frac{\varepsilon_{1} 2^{\alpha} k k^{\prime} m m^{\prime}}{\ell}\right)\left(\frac{\varepsilon_{1} \varepsilon_{2} 2^{\alpha+\beta} k k^{\prime} \ell \ell^{\prime}}{m}\right)=u\left(\frac{\ell^{\prime}}{k}\right)\left(\frac{k^{\prime}}{\ell}\right)\left(\frac{\ell^{\prime} k^{\prime}}{m}\right)\left(\frac{m^{\prime}}{k \ell}\right),
$$

avec

$$
u=u(k, \ell, m)=(-1)^{\vartheta(k) \vartheta(\ell)+\vartheta(k) \vartheta(m)+\vartheta(m) \vartheta(\ell)}\left(\frac{\varepsilon_{2} 2^{\beta}}{k m}\right)\left(\frac{\varepsilon_{1} 2^{\alpha}}{\ell m}\right) .
$$

Un calcul simple fournit le résultat suivant.

Lemme 4.1. - Lorsque $(k, \ell) \equiv\left(k_{0}, \ell_{0}\right)(\bmod 8)$ et $\left(\varepsilon_{1} k_{0} m, \varepsilon_{2} \ell_{0} m\right) \in E_{\alpha, \beta}$, on a

$$
u(k, \ell, m)=(-1)^{\vartheta\left(\varepsilon_{1} k_{0} m\right) \vartheta\left(\varepsilon_{2} \ell_{0} m\right)+\vartheta\left(\varepsilon_{1}\right) \vartheta\left(\varepsilon_{2}\right)+\vartheta(m)} .
$$

Démonstration. - Nous avons toujours

$$
\left(\frac{2^{\beta}}{k_{0} m}\right)\left(\frac{2^{\alpha}}{\ell_{0} m}\right)=1
$$

En effet, le cas $(\alpha, \beta)=(0,0)$ étant trivial, regardons le cas $(\alpha, \beta)=(1,0)$. Il découle de (4.1) que $\varepsilon_{2} \ell_{0} m \equiv 1(\bmod 8)$ ce qui montre la formule dans ce cas. Les raisonnements 
sont identiques pour $(\alpha, \beta)=(0,1)$ ou $(1,1)$. Nous avons donc bien l'expression attendue. Comme

nous avons

$$
\left(\frac{\varepsilon_{2}}{k m}\right)\left(\frac{\varepsilon_{1}}{\ell m}\right)=(-1)^{\vartheta\left(k_{0} m\right) \vartheta\left(\varepsilon_{2}\right)+\vartheta\left(\ell_{0} m\right) \vartheta\left(\varepsilon_{1}\right)},
$$

$$
\begin{aligned}
& u(k, \ell, m)=(-1)^{\vartheta(k) \vartheta(\ell)+\vartheta(k) \vartheta(m)+\vartheta(m) \vartheta(\ell)+\vartheta\left(k_{0} m\right) \vartheta\left(\varepsilon_{2}\right)+\vartheta\left(\ell_{0} m\right) \vartheta\left(\varepsilon_{1}\right)} \\
& =(-1)^{\vartheta\left(\varepsilon_{1} k_{0} m\right) \vartheta\left(\varepsilon_{2} \ell_{0} m\right)+\vartheta\left(\varepsilon_{1}\right) \vartheta\left(\varepsilon_{2}\right)+\vartheta(m)},
\end{aligned}
$$

ce qui fournit le résultat recherché.

Nous reprenons la démarche développée dans [4]. Pour cela, nous considérons

$$
V=(\log P)^{B},
$$

où $B$ est un paramètre qui sera choisi suffisamment grand en fonction de la valeur de $A$ prise dans les applications du Corollaire 3.2 .

La contribution à $T(P ; \alpha, \beta, \varepsilon)$ des couples d'entiers $(a, b)$ tels que $m m^{\prime}>V$ est $\ll P^{2} / V$. Dorénavant, nous nous restreignons au cas $m m^{\prime} \leqslant V$.

La contribution à $T(P ; \alpha, \beta, \varepsilon)$ des couples d'entiers $(a, b)$ tels que $k \leqslant V$ et $k^{\prime} \leqslant V$ est $\ll P V^{2} \log P$. De même lorsque $\ell \leqslant V$ et $\ell^{\prime} \leqslant V$.

Grâce au Lemme 3.3, du fait de la présence du facteur $\left(\frac{\ell^{\prime}}{k}\right)$, la contribution du cas $\ell^{\prime}>V$ et $k>V$ est

$$
\begin{aligned}
& \ll \sum_{m m^{\prime} \leqslant V} \sum_{\ell \leqslant P /\left(m m^{\prime} V\right)} \sum_{k^{\prime} \leqslant P /\left(m m^{\prime} V\right)} \frac{P^{2}}{m m^{\prime} k^{\prime} \ell}\left\{\left(\frac{P}{m m^{\prime} \ell}\right)^{-\frac{1}{6}}+\left(\frac{P}{m m^{\prime} k^{\prime}}\right)^{-\frac{1}{6}}\right\}(\log P)^{\frac{7}{6}} \\
& \ll P^{2} V^{-\frac{1}{6}}(\log P)^{\frac{13}{6}}(\log V)^{2},
\end{aligned}
$$

ce qui suffit lorsque $B>25$. Nous avons la même majoration lorsque $\ell>V$ et $k^{\prime}>V$ grâce à la présence du facteur $\left(\frac{k^{\prime}}{\ell}\right)$.

Il nous reste à traiter le cas $k, \ell \leqslant V$ ou $k^{\prime}, \ell^{\prime} \leqslant V$. Dans le premier cas, nous sommes amenés à considérer lorsque $\left(\varepsilon_{1} m m^{\prime} k k_{0}^{\prime}, \varepsilon_{2} m m^{\prime} \ell \ell_{0}^{\prime}\right) \in E_{\alpha, \beta}$ la somme

$$
T_{k, \ell}\left(k_{0}^{\prime}, \ell_{0}^{\prime}, m, m^{\prime}\right)=\sum_{\substack{\left(k^{\prime}, \ell^{\prime}\right) \in \mathbb{N}^{2} \\ k^{\prime} \leqslant P /\left(2^{\alpha} m m^{\prime} k\right), \ell^{\prime} \leqslant P /\left(2^{\beta} m m^{\prime} \ell\right) \\\left(k^{\prime}, \ell^{\prime}\right)=\left(k^{\prime} \ell^{\prime}, m m^{\prime} k \ell\right)=1 \\\left(k^{\prime}, \ell^{\prime}\right) \equiv\left(k_{0}^{\prime}, \ell_{0}^{\prime}\right)(\bmod 8)}} \frac{\mu^{2}\left(k^{\prime}\right)}{2^{\omega\left(k^{\prime}\right)}} \frac{\mu^{2}\left(\ell^{\prime}\right)}{2^{\omega\left(\ell^{\prime}\right)}}\left(\frac{\ell^{\prime}}{k m}\right)\left(\frac{k^{\prime}}{\ell m}\right),
$$

alors que, dans le deuxième cas, nous estimerons lorsque $\left(\varepsilon_{1} m m^{\prime} k_{0} k^{\prime}, \varepsilon_{2} m m^{\prime} \ell_{0} \ell^{\prime}\right) \in E_{\alpha, \beta}$ la somme

$$
T_{k^{\prime}, \ell^{\prime}}^{\prime}\left(k_{0}, \ell_{0}, m, m^{\prime}\right)=\sum_{\substack{(k, \ell) \in \mathbb{N}^{2} \\ k \leqslant P /\left(2^{\alpha} m m^{\prime} k^{\prime}\right), \ell \leqslant P /\left(2^{\beta} m m^{\prime} \ell^{\prime}\right) \\\left(, \ell, \ell=\left(k \ell, m m^{\prime} k^{\prime} \ell^{\prime}\right)=1 \\(k, \ell) \equiv\left(k 0, \ell_{0}\right)(\bmod 8)\right.}} \frac{\mu^{2}(k)}{2^{\omega(k)}} \frac{\mu^{2}(\ell)}{2^{\omega(\ell)}}\left(\frac{\ell^{\prime} m^{\prime}}{k}\right)\left(\frac{k^{\prime} m^{\prime}}{\ell}\right) .
$$

En effet $u(k, \ell, m)=u\left(k_{0}, \ell_{0}, m\right)$, lorsque $(k, \ell) \equiv\left(k_{0}, \ell_{0}\right)(\bmod 8)$, ne dépend pas de $(k, \ell)$. 
Nous avons

$$
T_{k, \ell}\left(k_{0}^{\prime}, \ell_{0}^{\prime}, m, m^{\prime}\right)=Q\left(\frac{P}{2^{\alpha} m m^{\prime} k}, \frac{P}{2^{\beta} m m^{\prime} \ell} ; k_{0}^{\prime}, \ell_{0}^{\prime}, m^{\prime} k, m^{\prime} \ell, 8, \ell m, k m, \chi_{\ell m}, \chi_{k m}\right),
$$

où $\chi_{n}(\cdot)=(\dot{\dot{n}})$. Cette somme peut donc être estimée grâce au Corollaire [3.2. Nous obtenons

$$
T_{k, \ell}\left(k_{0}^{\prime}, \ell_{0}^{\prime}, m, m^{\prime}\right)=\frac{\mathbf{1}_{k=\ell=m=1}}{2^{2+\alpha+\beta} \pi^{3}} \frac{\varphi_{2}\left(m^{\prime}\right)}{m^{\prime 2}} \frac{P^{2}}{\log P}\left\{1+O\left(\frac{\log \left(2 m^{\prime}\right)}{\log P}\right)\right\}+O\left(\frac{P^{2} \tau(k l)}{(\log P)^{A}}\right),
$$

où nous avons utilisé la formule $c\left(m^{\prime}, m^{\prime}, 8,1,1\right)=4 \varphi_{2}\left(m^{\prime}\right) / \pi^{3}$.

De même, posant

$$
u^{\prime}\left(k_{0}, \ell_{0}, m^{\prime}\right)=(-1)^{\vartheta\left(\ell_{0}\right) \vartheta\left(k^{\prime} m^{\prime}\right)+\vartheta\left(k_{0}\right) \vartheta\left(\ell^{\prime} m^{\prime}\right)},
$$

nous obtenons grâce à (2.5)

$$
\begin{aligned}
T_{k^{\prime}, \ell^{\prime}}^{\prime}\left(k_{0}, \ell_{0}, m, m^{\prime}\right) & =u^{\prime}\left(k_{0}, \ell_{0}, m^{\prime}\right) T_{k^{\prime}, \ell^{\prime}}\left(k_{0}, \ell_{0}, m^{\prime}, m\right) \\
& =\frac{\mathbf{1}_{k^{\prime}=\ell^{\prime}=m^{\prime}=1}}{2^{2+\alpha+\beta} \pi^{3}} \frac{\varphi_{2}(m)}{m^{2}} \frac{P^{2}}{\log P}\left\{1+O\left(\frac{\log (2 m)}{\log P}\right)\right\}+O\left(\frac{P^{2} \tau\left(k^{\prime} \ell^{\prime}\right)}{(\log P)^{A}}\right) .
\end{aligned}
$$

Enfin, nous en déduisons

$$
\begin{aligned}
T(P, \alpha, \beta, \varepsilon)= & \frac{1}{\pi^{3}}\left(\sum_{\substack{m \in \mathbb{N} \\
2 \nmid m}} \frac{\mu^{2}(m) \varphi_{2}(m)}{2^{\omega(m)} m^{2}} \tau_{\alpha, \beta}(m)\right) \frac{P^{2}}{\log P}\left\{1+O\left(\frac{1}{\log P}\right)\right\} \\
& +O\left(P^{2}(\log V)^{3}\left\{\frac{V^{3}}{(\log P)^{A}}+\frac{(\log P)^{\frac{13}{6}}}{V^{\frac{1}{6}}}\right\}\right),
\end{aligned}
$$

avec

$$
\tau_{\alpha, \beta}(m)=\frac{\# E_{\alpha, \beta}}{2^{\alpha+\beta}}+\sum_{\begin{array}{c}
\varepsilon_{1}, \varepsilon_{2} \in\{ \pm 1\} \\
\left(k_{0}, \ell_{0}\right) \in(\mathbb{Z} / 8 \mathbb{Z})^{2} \\
\left(\varepsilon_{1} k_{0} m, \varepsilon_{2} \ell_{0} m\right) \in E_{\alpha, \beta}(\bmod 8)
\end{array}} \frac{u\left(k_{0}, \ell_{0}, m\right)}{2^{2+\alpha+\beta}},
$$

où $u$ a été défini en (4.4).

Grâce à (4.1), nous avons

$$
\sum_{(\alpha, \beta) \in\{0,1\}^{2}} \frac{\# E_{\alpha, \beta}}{2^{\alpha+\beta}}=15 .
$$

Le Lemme 4.1 et les égalités (4.1) fournissent ainsi

$$
\begin{aligned}
\sum_{(\alpha, \beta) \in\{0,1\}^{2}} \tau_{\alpha, \beta}(m) & =15+\sum_{(\alpha, \beta) \in\{0,1\}^{2}} \sum_{\substack{\varepsilon_{1}, \varepsilon_{2} \in\{ \pm 1\} \\
\left(u_{0}, v_{0}\right) \in E_{\alpha, \beta}(\bmod 8)}} \frac{(-1)^{\vartheta\left(\varepsilon_{1}\right) \vartheta\left(\varepsilon_{2}\right)}}{2^{2+\alpha+\beta}}(-1)^{\vartheta\left(u_{0}\right) \vartheta\left(v_{0}\right)+\vartheta(m)} \\
& =15+(-1)^{\vartheta(m)} \sum_{\substack{(\alpha, \beta) \in\{0,1\}^{2} \\
2^{1+\alpha+\beta}}} \sum_{\left(u_{0}, v_{0}\right) \in E_{\alpha, \beta}(\bmod 8)}(-1)^{\vartheta\left(u_{0}\right) \vartheta\left(v_{0}\right)} \\
& =15+5(-1)^{\vartheta(m)} .
\end{aligned}
$$


Un simple calcul fournit

$$
\sum_{\substack{m \in \mathbb{N} \\ 2 \nmid m}} \frac{z^{\vartheta(m)} \mu^{2}(m) \varphi_{2}(m)}{2^{\omega(m)} m^{2}}=\prod_{p>2}\left(1+\frac{z^{\vartheta(p)}}{2 p(p+1)}\right), \quad(z \in\{ \pm 1\}) .
$$

En choisissant $A=81$ and $B=26$, nous obtenons

$$
T(P)=C \frac{P^{2}}{\log P}\left\{1+O\left(\frac{1}{\log P}\right)\right\},
$$

où

$$
C=\frac{15}{\pi^{3}} \prod_{p>2}\left(1+\frac{1}{2 p(p+1)}\right)+\frac{5}{\pi^{3}} \prod_{p>2}\left(1+\frac{(-1)^{\vartheta(p)}}{2 p(p+1)}\right) .
$$

À partir de (2.3), il vient ainsi

$$
N_{1}(P)=\frac{6 C}{\pi^{2}} \frac{P^{3}}{\log P}\left\{1+O\left(\frac{1}{\log P}\right)\right\} .
$$

\section{5. Étude de $N_{2}(P)$}

Notre objectif dans cette section est d'estimer la somme

$$
N_{2}(P)=\sum_{(a, b, c) \in S(P)} f(a, b) h(a, b, c) .
$$

Les calculs sont plus compliqués que pour l'estimation de $N_{1}(P)$ mais relèvent des mêmes méthodes.

Lorsque $f(a, b) \neq 0$, nous aurons besoin d'une expression simple de la fonction $h(a, b, c)$ définie en (2.1). Comme $(c, b)_{p}=1$ pour tout nombre premier impair $p$ ne divisant pas $b c$, nous avons

$$
h(a, b, c)=\prod_{\substack{p \mid 2 b c \\ a \notin \mathbb{Q}_{p}^{* 2}}}(c, b)_{p}
$$

Rappelons la définition (1.2) de $S$. Nous paramétrons les $(a, b, c) \in S$ par

$$
a=\varepsilon_{1} 2^{\alpha} d_{0} d_{12} d_{13} a^{\prime}, \quad b=\varepsilon_{2} 2^{\beta} d_{0} d_{12} d_{23} b^{\prime}, \quad c=2^{\gamma} d_{0} d_{13} d_{23} c^{\prime},
$$

avec $d_{i j}, d_{0}, a^{\prime}, b^{\prime}, c^{\prime}$ des nombres impairs, $\alpha, \beta, \gamma \in\{0,1\}$ et les conditions de coprimalité

$$
\begin{aligned}
& \left(d_{12}, d_{13}\right)=1, \quad\left(d_{12}, d_{23}\right)=1, \quad\left(d_{13}, d_{23}\right)=1, \\
& \left(a^{\prime} b^{\prime} c^{\prime}, d_{12} d_{13} d_{23}\right)=\left(a^{\prime}, b^{\prime} c^{\prime}\right)=\left(b^{\prime}, c^{\prime}\right)=1 .
\end{aligned}
$$

Nous écrivons $h(a, b, c)=h_{1}(a, b, c) h_{2}(a, b, c)$, avec $h_{1}(a, b, c)$ le produit sur les $p$ impairs et la quantité $h_{2}(a, b, c)$ désignant le facteur lié à $p=2$. Les deux résultats suivants concernent leur calcul explicite. 
Lemme 5.1. - Lorsque $(a, b, c) \in S$ et $f(a, b) \neq 0$, on $a$

$$
h_{1}(a, b, c)=\frac{1}{2^{\omega\left(c^{\prime}\right)}} \sum_{n n^{\prime} n^{\prime \prime} n^{\prime \prime \prime}=c^{\prime}} \tilde{u}\left(\frac{a^{\prime}}{n n^{\prime \prime}}\right)\left(\frac{b^{\prime}}{n^{\prime} n^{\prime \prime} d_{0} d_{13}}\right) \text {, }
$$

où

$$
\begin{aligned}
\tilde{u} & =\tilde{u}\left(n, n^{\prime}, n^{\prime \prime}, n^{\prime \prime \prime}, d_{0}, d_{12}, d_{13}, d_{23}\right) \\
& =\mu\left(n^{\prime \prime}\right)(-1)^{\vartheta\left(d_{0} d_{12}\right) \vartheta\left(d_{0} d_{13} n n^{\prime}\right)}\left(\frac{\varepsilon_{1} 2^{\alpha} d_{13}}{n n^{\prime \prime}}\right)\left(\frac{\varepsilon_{2} 2^{\beta} d_{23}}{n^{\prime} n^{\prime \prime}}\right)\left(\frac{\varepsilon_{2} 2^{\beta}}{d_{0} d_{13}}\right)\left(\frac{2^{\gamma} n^{\prime \prime} n^{\prime \prime \prime}}{d_{0} d_{12}}\right)\left(\frac{d_{23}}{d_{12} d_{13}}\right) .
\end{aligned}
$$

Démonstration. - Lorsque $a, b, c$ sont sans facteur carré et $p \mid a$, alors $a \notin \mathbb{Q}_{p}^{* 2}$. De plus, lorsque $p \nmid a$ et $p \mid b$, le fait que $f(a, b) \neq 0$ implique que $a$ est un carré dans $\mathbb{Q}_{p}^{*}$ ce qui est exclu. Lorsque $p \nmid a$, nous nous restreignons à $p \nmid b$ et donc $p \mid c$ et $\left(\frac{a}{p}\right)=-1$. Ainsi d'après (2.6), nous avons $(c, b)_{p}=\left(\frac{b}{p}\right)$. Il vient

$$
h_{1}(a, b, c)=\prod_{\substack{p \mid(b c, a) \\ p>2}}(c, b)_{p} \prod_{\substack{p \mid c \\ p \nmid 2 a b}} \frac{1}{2}\left\{1+\left(\frac{a}{p}\right)+\left(\frac{b}{p}\right)-\left(\frac{a b}{p}\right)\right\} .
$$

En développant le produit, nous obtenons

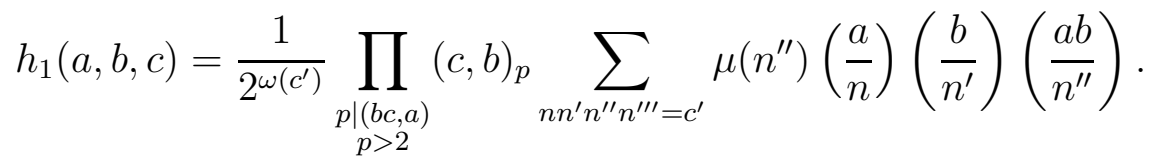

De plus, nous avons

$$
\begin{aligned}
\prod_{\substack{p \mid(b c, a) \\
p>2}}(c, b)_{p} & =\prod_{p \mid d_{0}}\left(\frac{-1}{p}\right)\left(\frac{b c / p^{2}}{p}\right) \prod_{\substack{p \mid(a, b) \\
p \nmid 2 c}}\left(\frac{c}{p}\right) \prod_{\substack{p \mid(a, c) \\
p \nmid 2 b}}\left(\frac{b}{p}\right) \\
& =\left(\frac{-1}{d_{0}}\right)\left(\frac{b c / d_{0}^{2}}{d_{0}}\right) \prod_{\substack{p \mid(a, b) \\
p \nmid 2 c}}\left(\frac{c}{p}\right) \prod_{\substack{p \mid(a, c) \\
p \nmid 2 b}}\left(\frac{b}{p}\right) .
\end{aligned}
$$

En utilisant les notations (5.1), nous obtenons

$$
\begin{aligned}
\prod_{\substack{p \mid(b c, a) \\
p>2}}(c, b)_{p} & =\left(\frac{-1}{d_{0}}\right)\left(\frac{\varepsilon_{2} 2^{\beta+\gamma} b^{\prime} c^{\prime} d_{12} d_{13}}{d_{0}}\right)\left(\frac{2^{\gamma} d_{0} d_{13} d_{23} c^{\prime}}{d_{12}}\right)\left(\frac{\varepsilon_{2} 2^{\beta} d_{0} d_{12} d_{23} b^{\prime}}{d_{13}}\right) \\
& =\left(\frac{\varepsilon_{2} 2^{\beta} b^{\prime}}{d_{0} d_{13}}\right)\left(\frac{2^{\gamma} c^{\prime}}{d_{0} d_{12}}\right)\left(\frac{d_{23}}{d_{12} d_{13}}\right)(-1)^{\vartheta\left(d_{0} d_{12}\right) \vartheta\left(d_{0} d_{13}\right)},
\end{aligned}
$$

puisque la loi de réciprocité quadratique (2.5) fournit

$$
\left(\frac{-1}{d_{0}}\right)\left(\frac{d_{12} d_{13}}{d_{0}}\right)\left(\frac{d_{0} d_{13}}{d_{12}}\right)\left(\frac{d_{0} d_{12}}{d_{13}}\right)=(-1)^{\vartheta\left(d_{0} d_{12}\right) \vartheta\left(d_{0} d_{13}\right)} .
$$


Cela implique ainsi

$$
\begin{aligned}
h_{1}(a, b, c)= & \frac{1}{2^{\omega\left(c^{\prime}\right)}} \sum_{n n^{\prime} n^{\prime \prime} n^{\prime \prime \prime}=c^{\prime}} \mu\left(n^{\prime \prime}\right)\left(\frac{a}{n}\right)\left(\frac{b}{n^{\prime}}\right)\left(\frac{a b}{n^{\prime \prime}}\right) \\
& \times\left(\frac{\varepsilon_{2} 2^{\beta} b^{\prime}}{d_{0} d_{13}}\right)\left(\frac{2^{\gamma} c^{\prime}}{d_{0} d_{12}}\right)\left(\frac{d_{23}}{d_{12} d_{13}}\right)(-1)^{\vartheta\left(d_{0} d_{12}\right) \vartheta\left(d_{0} d_{13}\right)} .
\end{aligned}
$$

Puis, prenant $\tilde{u}$ comme dans l'énoncé du lemme, nous obtenons le résultat attendu après calcul de $\left(\frac{n n^{\prime}}{d_{0} d_{12}}\right)\left(\frac{d_{0} d_{12}}{n n^{\prime}}\right)$ par la loi de réciprocité quadratique (2.5).

Lemme 5.2. - Lorsque $(a, b, c) \in S, f(a, b) \neq 0$ et

$$
(a, b, c)=\left(2^{\alpha} u, 2^{\beta} v, 2^{\gamma} w\right)
$$

avec $u, v, w$ impair, on a

$$
h_{2}(a, b, c)= \begin{cases}1, & \text { si } a \equiv 1(\bmod 8), \\ 1, & \text { si } 2 \mid a \text { et } b \equiv 1(\bmod 8) \\ (-1)^{\vartheta(v) \vartheta(w)+\frac{\gamma\left(v^{2}-1\right)}{8}+\frac{w^{2}-1}{8},}, & \text { si } 2 \mid(a, b) \text { et } u v \equiv 1(\bmod 8), \\ (-1)^{\vartheta(b) \vartheta(w)+\frac{\gamma\left(b^{2}-1\right)}{8},} & \text { si } a \equiv 3,5,7(\bmod 8) \text { et } 1 \in\{b, a b\}(\bmod 8), \\ 0, & \text { sinon. }\end{cases}
$$

Démonstration. - Si $a \equiv 1(\bmod 8)$ alors $a \in \mathbb{Q}_{2}^{* 2}$ et ainsi $h_{2}(a, b, c)=1$. Si $2 \mid a$ et $2 \nmid b$, alors $f(a, b) \neq 0$ implique $b \equiv 1(\bmod 8)$. La formule (2.7) implique encore $h_{2}(a, b, c)=1$. Si $2 \mid(a, b)$, alors $f(a, b) \neq 0$ implique $a b \equiv 4(\bmod 32)$. Donc la formule (2.7) implique le résultat. Si $a \equiv 3,5,7(\bmod 8)$, alors $f(a, b) \neq 0$ implique que $b$ est impair et que $b$ ou $a b$ est congru à $1(\bmod 8)$. Lorsque $c=2^{\gamma} w$, la formule (2.7) implique le résultat.

Il est clair que la valeur de $h_{2}(a, b, c)$ ne dépend que de la valeur modulo 8 de $(u, v, w)$ et des valuations 2 -adiques $\alpha, \beta, \gamma$.

Avec les notations (5.1), nous avons

$$
a /(a, b)=\varepsilon_{1} 2^{\alpha-\min \{\alpha, \beta\}} a^{\prime} d_{13}, \quad b /(a, b)=\varepsilon_{2} 2^{\beta-\min \{\alpha, \beta\}} b^{\prime} d_{23}, \quad(a, b)=2^{\min \{\alpha, \beta\}} d_{0} d_{12} .
$$

Dans la sommation (4.2), nous remplaçons $\left(k, k^{\prime}, \ell, \ell^{\prime}, m, m^{\prime}\right)$ par

$$
\left(k k_{13}, k^{\prime} k_{13}^{\prime}, \ell \ell_{23}, \ell^{\prime} \ell_{23}^{\prime}, m_{0} m_{12}, m_{0}^{\prime} m_{12}^{\prime}\right)
$$

tels que

$$
k k^{\prime}=a^{\prime}, k_{13} k_{13}^{\prime}=d_{13}, \quad \ell \ell^{\prime}=b^{\prime}, \ell_{23} \ell_{23}^{\prime}=d_{23}, \quad m_{0} m_{0}^{\prime}=d_{0}, m_{12} m_{12}^{\prime}=d_{12} .
$$

Lorsque $\left(a 2^{-\alpha}, b 2^{-\beta}\right) \equiv\left(a_{0}, b_{0}\right)(\bmod 8)$ avec $\left(a_{0}, b_{0}\right) \in E_{\alpha, \beta}, \varepsilon_{1} a>0, \varepsilon_{2} b>0$ où $\varepsilon \in\{ \pm 1\}^{2}$, la formule (4.2) s'écrit aussi

$$
\begin{aligned}
& f(a, b)=\frac{1}{2^{\omega\left(2^{-\alpha-\beta} a b\right)}} \sum\left(\frac{\varepsilon_{2} 2^{\beta} \ell \ell^{\prime} \ell_{23} \ell_{23}^{\prime} m_{0} m_{0}^{\prime} m_{12} m_{12}^{\prime}}{k k_{13}}\right) \\
& \times\left(\frac{\varepsilon_{1} 2^{\alpha} k k^{\prime} k_{13} k_{13}^{\prime} m_{0} m_{0}^{\prime} m_{12} m_{12}^{\prime}}{\ell \ell_{23}}\right)\left(\frac{\varepsilon_{1} \varepsilon_{2} 2^{\alpha+\beta} k k^{\prime} k_{13} k_{13}^{\prime} \ell \ell^{\prime} \ell_{23} \ell_{23}^{\prime}}{m_{0} m_{12}}\right) .
\end{aligned}
$$


avec $a=\varepsilon_{1} 2^{\alpha} k k^{\prime} k_{13} k_{13}^{\prime} m_{0} m_{0}^{\prime} m_{12} m_{12}^{\prime}$ et $b=\varepsilon_{2} 2^{\beta} \ell \ell^{\prime} \ell_{23} \ell_{23}^{\prime} m_{0} m_{0}^{\prime} m_{12} m_{12}^{\prime}$ satisfaisant (5.2). La formule (4.3) fournit alors

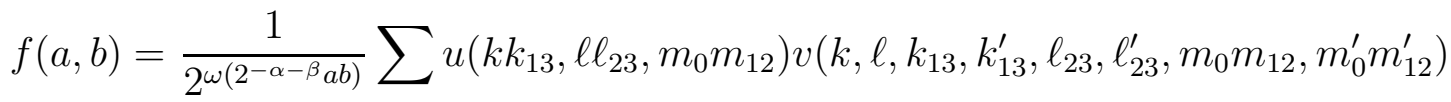

$$
\begin{aligned}
& \times\left(\frac{\ell^{\prime}}{k}\right)\left(\frac{k^{\prime}}{\ell}\right)\left(\frac{k}{\ell_{23}^{\prime} m_{0}^{\prime} m_{12}^{\prime}}\right)\left(\frac{k^{\prime}}{\ell_{23} m_{0} m_{12}}\right)\left(\frac{\ell}{k_{13}^{\prime} m_{0}^{\prime} m_{12}^{\prime}}\right)\left(\frac{\ell^{\prime}}{k_{13} m_{0} m_{12}}\right),
\end{aligned}
$$

avec

$$
\begin{aligned}
v & =v\left(k, \ell, k_{13}, k_{13}^{\prime}, \ell_{23}, \ell_{23}^{\prime}, m_{0} m_{12}, m_{0}^{\prime} m_{12}^{\prime}\right) \\
& =\left(\frac{\ell_{23}^{\prime}}{k_{13} m_{0} m_{12}}\right)\left(\frac{k_{13}^{\prime}}{\ell_{23} m_{0} m_{12}}\right)\left(\frac{m_{0}^{\prime} m_{12}^{\prime}}{k_{13} \ell_{23}}\right)(-1)^{\vartheta\left(k_{13}^{\prime} m_{0}^{\prime} m_{12}^{\prime}\right) \vartheta(\ell)+\vartheta\left(\ell_{23}^{\prime} m_{0}^{\prime} m_{12}^{\prime}\right) \vartheta(k) .}
\end{aligned}
$$

Le paramétrage (5.2) fournit aussi

$$
h_{1}(a, b, c)=\frac{1}{2^{\omega\left(c^{\prime}\right)}} \sum_{n n^{\prime} n^{\prime \prime} n^{\prime \prime \prime}=c^{\prime}} \tilde{u}\left(\frac{k k^{\prime}}{n n^{\prime \prime}}\right)\left(\frac{\ell \ell^{\prime}}{n^{\prime} n^{\prime \prime} m_{0} m_{0}^{\prime} k_{13} k_{13}^{\prime}}\right),
$$

grâce au Lemme 5.1 .

Lorsque $\left(a 2^{-\alpha}, b 2^{-\beta}, c 2^{-\gamma}\right) \equiv\left(u_{0}, v_{0}, w_{0}\right)(\bmod 8)$ avec $\left(u_{0}, v_{0}\right) \in E_{\alpha, \beta}, \varepsilon_{1} a>0, \varepsilon_{2} b>0$ où $\varepsilon \in\{ \pm 1\}^{2}$, nous devons donc sommer le terme

$$
\begin{aligned}
f(a, b) h(a, b, c)= & \sum \frac{\tilde{u}_{1}}{2^{\omega\left(2^{-\alpha-\beta-\gamma} a b c\right)}}\left(\frac{k k^{\prime}}{n n^{\prime \prime}}\right)\left(\frac{\ell \ell^{\prime}}{n^{\prime} n^{\prime \prime} m_{0} m_{0}^{\prime} k_{13} k_{13}^{\prime}}\right)\left(\frac{\ell^{\prime}}{k}\right)\left(\frac{k^{\prime}}{\ell}\right) \\
& \times\left(\frac{k}{\ell_{23}^{\prime} m_{0}^{\prime} m_{12}^{\prime}}\right)\left(\frac{k^{\prime}}{\ell_{23} m_{0} m_{12}}\right)\left(\frac{\ell}{k_{13}^{\prime} m_{0}^{\prime} m_{12}^{\prime}}\right)\left(\frac{\ell^{\prime}}{k_{13} m_{0} m_{12}}\right) \\
= & \sum \frac{\tilde{u}_{1}}{2^{\omega\left(2^{-\alpha-\beta-\gamma} a b c\right)}}\left(\frac{k k^{\prime}}{n n^{\prime \prime}}\right)\left(\frac{\ell \ell^{\prime}}{n^{\prime} n^{\prime \prime}}\right)\left(\frac{\ell^{\prime}}{k}\right)\left(\frac{k^{\prime}}{\ell}\right) \\
& \times\left(\frac{k}{\ell_{23}^{\prime} m_{0}^{\prime} m_{12}^{\prime}}\right)\left(\frac{k^{\prime}}{\ell_{23} m_{0} m_{12}}\right)\left(\frac{\ell}{k_{13} m_{0} m_{12}^{\prime}}\right)\left(\frac{\ell^{\prime}}{k_{13}^{\prime} m_{0}^{\prime} m_{12}}\right),
\end{aligned}
$$

avec des sommations sur les entiers satisfaisant (5.2) et $c^{\prime}=n n^{\prime} n^{\prime \prime} n^{\prime \prime \prime}$, où

$$
\begin{aligned}
\tilde{u}_{1}= & \tilde{u}_{1}\left(k, k^{\prime}, \ell, \ell^{\prime}, k_{13}, k_{13}^{\prime}, \ell_{23}, \ell_{23}^{\prime}, m_{0}, m_{0}^{\prime}, m_{12}, m_{12}^{\prime}\right) \\
= & u\left(k k_{13}, \ell \ell_{23}, m_{0} m_{12}\right) v\left(k, \ell, k_{13}, k_{13}^{\prime}, \ell_{23}, \ell_{23}^{\prime}, m_{0} m_{12}, m_{0}^{\prime} m_{12}^{\prime}\right) \\
& \times \tilde{u}\left(n, n^{\prime}, n^{\prime \prime}, n^{\prime \prime \prime}, m_{0} m_{0}^{\prime}, m_{12} m_{12}^{\prime}, k_{13} k_{13}^{\prime}, \ell_{23} \ell_{23}^{\prime}\right) h_{2}(a, b, c) .
\end{aligned}
$$

Ici, nous avons

$$
\begin{aligned}
& a=\varepsilon_{1} 2^{\alpha} k k^{\prime} k_{13} k_{13}^{\prime} m_{0} m_{0}^{\prime} m_{12} m_{12}^{\prime}, \\
& b=\varepsilon_{2} 2^{\beta} \ell \ell^{\prime} \ell_{23} \ell_{23}^{\prime} m_{0} m_{0}^{\prime} m_{12} m_{12}^{\prime}, \\
& c=2^{\gamma} n n^{\prime} n^{\prime \prime} n^{\prime \prime \prime} k_{13} k_{13}^{\prime} \ell_{23} \ell_{23}^{\prime} m_{0} m_{0}^{\prime} .
\end{aligned}
$$


La sommation sur $(a, b, c)$ s'opère de la même manière que pour $N_{1}(B)$. Quitte à négliger une contribution englobée dans le terme d'erreur, nous pouvons supposer que

$$
m_{0} m_{0}^{\prime} m_{12} m_{12}^{\prime} \leqslant V, \quad k_{13} k_{13}^{\prime} \leqslant V, \quad \ell_{23} \ell_{23}^{\prime} \leqslant V
$$

avec $V=(\log P)^{B}$. Puis en appliquant le Lemme 3.3 du fait du facteur $\left(\frac{\ell^{\prime}}{k}\right)\left(\frac{k^{\prime}}{\ell}\right)$, nous pouvons nous restreindre au cas $k, \ell \leqslant V$ ou $k^{\prime}, \ell^{\prime} \leqslant V$. De même, grâce au Lemme 3.3 et le facteur $\left(\frac{k k^{\prime}}{n n^{\prime \prime}}\right)\left(\frac{\ell \ell^{\prime}}{n^{\prime} n^{\prime \prime}}\right)$, nous pouvons désormais supposer que $n, n^{\prime}, n^{\prime \prime} \leqslant V$.

Le facteur $\tilde{u}_{1}$ ne dépend pas de $k, \ell, k^{\prime}, \ell^{\prime}$ mais seulement de leur valeur modulo 8. En fixant $\left(k^{\prime}, \ell^{\prime}\right) \equiv\left(k_{0}^{\prime}, \ell_{0}^{\prime}\right)(\bmod 8)$, la somme sur $\left(k^{\prime}, \ell^{\prime}\right)$ lorsque $k, \ell \leqslant V$ à estimer est

$$
Q\left(\frac{P}{2^{\alpha} m_{0} m_{0}^{\prime} m_{12} m_{12}^{\prime} k k_{13} k_{13}^{\prime}}, \frac{P}{2^{\beta} m_{0} m_{0}^{\prime} m_{12} m_{12}^{\prime} \ell \ell_{23} \ell_{23}^{\prime}} ; k_{0}^{\prime}, \ell_{0}^{\prime}, \mathbf{d}, 8, \mathbf{q}, \chi_{q_{1}}, \chi_{q_{2}}\right),
$$

avec

$$
\begin{aligned}
q_{1} & =\ell_{23} m_{0} m_{12} n n^{\prime \prime} \ell, & q_{2} & =k_{13}^{\prime} m_{0}^{\prime} m_{12} n^{\prime} n^{\prime \prime} k, \\
d_{1} & =n^{\prime} n^{\prime \prime \prime} m_{0}^{\prime} m_{12}^{\prime} k k_{13} k_{13}^{\prime} \ell_{23}^{\prime}, & & d_{2}=n n^{\prime \prime \prime} m_{0} m_{12}^{\prime} k_{13} \ell \ell_{23} \ell_{23}^{\prime} .
\end{aligned}
$$

Respectivement, en fixant $(k, \ell) \equiv\left(k_{0}, \ell_{0}\right)(\bmod 8)$, la somme sur $(k, \ell)$ à estimer lorsque $k^{\prime}, \ell^{\prime} \leqslant V$ est

$$
Q\left(\frac{P}{2^{\alpha} m_{0} m_{0}^{\prime} m_{12} m_{12}^{\prime} k^{\prime} k_{13} k_{13}^{\prime}}, \frac{P}{2^{\beta} m_{0} m_{0}^{\prime} m_{12} m_{12}^{\prime} \ell^{\prime} \ell_{23} \ell_{23}^{\prime}} ; k_{0}, \ell_{0}, \mathbf{d}^{\prime}, 8, \mathbf{q}^{\prime}, \chi_{q_{1}^{\prime}}, \chi_{q_{2}^{\prime}}\right),
$$

avec

$$
\begin{array}{ll}
q_{1}^{\prime}=m_{0}^{\prime} m_{12}^{\prime} n n^{\prime \prime} \ell^{\prime} \ell_{23}^{\prime}, & q_{2}^{\prime}=k_{13} m_{0} m_{12}^{\prime} n^{\prime} n^{\prime \prime} k^{\prime}, \\
d_{1}^{\prime}=n^{\prime} n^{\prime \prime \prime} m_{0} m_{12} k^{\prime} k_{13} k_{13}^{\prime} \ell_{23}, & d_{2}^{\prime}=n n^{\prime \prime \prime} m_{0}^{\prime} m_{12} k_{13}^{\prime} \ell \ell_{23} \ell_{23}^{\prime} .
\end{array}
$$

La contribution principale provient du cas où les deux modules $q_{1}, q_{2}$ (resp. $q_{1}^{\prime}, q_{2}^{\prime}$ ) des caractères sommés sont égaux à un. Après cette sommation, il reste quatre variables à sommer $\left(n^{\prime \prime \prime}, k_{13}, \ell_{23}^{\prime}, m_{12}^{\prime}\right)$ (resp. $\left.\left(n^{\prime \prime \prime}, k_{13}^{\prime}, \ell_{23}, m_{12}\right)\right)$.

Compte tenu du Corollaire 3.2, dans le premier cas, le terme principal obtenu pour l'évaluation de (5.3) est

$$
\begin{gathered}
\mathbf{1}_{\substack{\ell_{23} m_{0} m_{12} n n^{\prime \prime} \ell=1 \\
k_{13}^{\prime} m_{0}^{\prime} m_{12} n^{\prime} n^{\prime \prime} k=1}} \frac{\tilde{u}_{2}\left(m_{12}^{\prime}\right)}{\pi^{3}} \frac{\mu^{2}\left(2 k_{13} \ell_{23}^{\prime}\right)}{2^{2+\alpha+\beta}} \frac{\varphi_{2}\left(k_{13} \ell_{23}^{\prime} m_{12}^{\prime}\right)}{m_{12}^{\prime 2} \ell_{23}^{\prime} k_{13}} \frac{\mu^{2}\left(n^{\prime \prime \prime}\right) \varphi_{2}\left(n^{\prime \prime \prime}\right)}{2^{\omega\left(n^{\prime \prime \prime} \ell_{23}^{\prime} k_{13} m_{12}^{\prime}\right)}\left(\frac{n^{\prime \prime \prime}}{m_{12}^{\prime}}\right)} \\
\times \frac{P^{2}}{\log P}\left\{1+O\left(\frac{\left(\log 2 \ell_{23}^{\prime} k_{13} m_{12}^{\prime}\right)^{\frac{3}{2}}}{\log P}\right)\right\}
\end{gathered}
$$

avec

$$
\tilde{u}_{2}\left(m_{12}^{\prime}\right)=(-1)^{\vartheta\left(m_{12}^{\prime}\right) \vartheta\left(k_{13}\right)}\left(\frac{m_{12}^{\prime}}{k_{13}}\right)\left(\frac{2^{\gamma} \ell_{23}^{\prime}}{m_{12}^{\prime}}\right) h_{2}\left(\varepsilon_{1} 2^{\alpha} k_{0}^{\prime} k_{13} m_{12}^{\prime}, \varepsilon_{2} 2^{\beta} \ell_{0}^{\prime} \ell_{23}^{\prime} m_{12}^{\prime}, 2^{\gamma} n^{\prime \prime \prime} k_{13} \ell_{23}^{\prime}\right) .
$$

Nous avons utilisé ici la relation $u\left(k_{13}, 1,1\right)\left(\frac{\varepsilon_{2} 2^{\beta}}{k_{13}}\right)=1$, issue de (4.4). 
Ensuite nous sommons les $n^{\prime \prime \prime} \leqslant P /\left(2^{\gamma} k_{13} \ell_{23}^{\prime}\right)$ congrus à $n_{0}^{\prime \prime \prime}(\bmod 8)$ avec $n_{0}^{\prime \prime \prime}$ impair, premiers à $2 k_{13} \ell_{23}^{\prime}$ en appliquant le Lemme 3.1 avec $\nu=2$. Nous obtenons un terme principal

$$
\mathbf{1}_{m_{12}^{\prime}=1} \frac{\tilde{u}_{2}(1)}{\pi^{3}} \frac{\mu^{2}\left(2 k_{13} \ell_{23}^{\prime}\right)}{2^{4+\alpha+\beta+\gamma}} \frac{\varphi_{2}\left(k_{13} \ell_{23}^{\prime}\right) c_{2}\left(2 k_{13} \ell_{23}^{\prime}\right)}{2^{\omega\left(k_{13} \ell_{23}^{\prime}\right)}\left(k_{13} \ell_{23}^{\prime}\right)^{2}} \frac{P^{3}}{(\log P)^{\frac{3}{2}}}\left\{1+O\left(\frac{\left(\log 2 \ell_{23}^{\prime} k_{13}\right)^{\frac{3}{2}}}{\log P}\right)\right\},
$$

avec $\tilde{u}_{2}(1)=h_{2}\left(\varepsilon_{1} 2^{\alpha} k_{0}^{\prime} k_{13}, \varepsilon_{2} 2^{\beta} \ell_{0}^{\prime} \ell_{23}^{\prime}, 2^{\gamma} n_{0}^{\prime \prime \prime} k_{13} \ell_{23}^{\prime}\right)$. Nous aurons besoin du lemme suivant.

Lemme 5.3. - Soient $z \in\{ \pm 1\}$ et

$$
\nu_{2}(z)=\sum_{(\alpha, \beta, \gamma) \in\{0,1\}^{3}} \sum_{\substack{\left(u_{0}, v_{0}\right) \in E_{\alpha, \beta}(\bmod 8) \\ w_{0} \in(\mathbb{Z} / 8 \mathbb{Z})^{*}}} \frac{z^{\vartheta\left(u_{0}\right) \vartheta\left(v_{0}\right)}}{2^{\alpha+\beta+\gamma}} h_{2}\left(2^{\alpha} u_{0}, 2^{\beta} v_{0}, 2^{\gamma} w_{0}\right) .
$$

Alors $\nu_{2}(z)=68$.

Démonstration. - Nous utilisons l'expression (4.1) pour les $E_{\alpha, \beta}$ et le Lemme 5.2 pour le calcul de $h_{2}$. La somme $\nu_{2}(z)$ se décompose sous la forme suivante

$$
= \begin{cases}36, & \text { si } \alpha=0, u_{0} \equiv 1(\bmod 8), \\ 12, & \text { si }(\alpha, \beta)=(1,0), v_{0} \equiv 1(\bmod 8), \\ 0, & \text { si }(\alpha, \beta)=(1,1), \\ 16, & \text { si }(\alpha, \gamma)=(0,0), u_{0} \equiv 3,5,7(\bmod 8), \\ 4, & \text { si }(\alpha, \gamma)=(0,1), u_{0} \equiv 3,5,7(\bmod 8) .\end{cases}
$$

Pour la troisième ligne, on a noté que

$$
\sum_{w_{0} \in(\mathbb{Z} / 8 \mathbb{Z})^{*}} z^{\vartheta\left(w_{0}\right)}(-1)^{\frac{w_{0}^{2}-1}{8}}=0,
$$

ce qui achève la démonstration.

Ensuite, avec la notation de $\$ 3$, nous avons

$$
\begin{aligned}
c_{2}(2 r) & =c_{2}(1) \prod_{p \mid 2 r}\left(1+\frac{1}{2(p+1)}\right)^{-1} \\
& =\frac{6}{7 \sqrt{\pi}} \prod_{p}\left(1+\frac{1}{2(p+1)}\right)\left(1-\frac{1}{p}\right)^{\frac{1}{2}} \prod_{p \mid r}\left(1+\frac{1}{2(p+1)}\right)^{-1},
\end{aligned}
$$

pour un impair $r$. Du Lemme 5.3, il découle la formule

$$
\sum_{(\alpha, \beta, \gamma) \in\{0,1\}^{3}} \sum_{\substack{\varepsilon_{1}, \varepsilon_{2} \in\{ \pm 1\} \\\left(\varepsilon_{1} k_{0}^{\prime} k_{13}, \varepsilon_{2}^{\prime} \ell_{\prime}^{\prime} \ell_{23}^{\prime}\right) \in E_{\alpha, \beta}(\bmod 8) \\ n_{0}^{\prime \prime \prime} \in(\mathbb{Z} / 8 \mathbb{Z})^{*}}} \frac{\tilde{u}_{2}(1)}{2^{4+\alpha+\beta+\gamma}}=\frac{\nu_{2}(1)}{4}=17 .
$$


Puis enfin, nous sommons sur $k_{13}$ et $\ell_{23}^{\prime}$. Nous avons

$$
\sum_{k_{13}, \ell_{23}^{\prime} \in \mathbb{N}} \frac{\mu^{2}\left(2 k_{13} \ell_{23}^{\prime}\right) \varphi_{2}\left(k_{13} \ell_{23}^{\prime}\right)}{2^{\omega\left(k_{13} \ell_{23}^{\prime}\right)}\left(k_{13} \ell_{23}^{\prime}\right)^{2}} \prod_{p \mid k_{13} \ell_{23}^{\prime}}\left(1+\frac{1}{2(p+1)}\right)^{-1}=\prod_{p>2}\left(1+\frac{1}{p\left(p+\frac{3}{2}\right)}\right) .
$$

Nous sommes donc amenés à une première contribution de (5.3) à $N_{2}(P)$ égale à

$$
17 \times \frac{6}{7 \pi^{\frac{7}{2}}} \times \frac{7}{8} \prod_{p} \frac{\left(1-\frac{1}{p}\right)^{\frac{1}{2}}}{\left(1+\frac{1}{p}\right)}\left(1+\frac{3}{2 p}+\frac{1}{p^{2}}\right) \frac{P^{3}}{(\log P)^{\frac{3}{2}}}\left\{1+O\left(\frac{1}{\log P}\right)\right\} .
$$

Nous passons maintenant à la deuxième contribution, liée à (5.4). Grâce au Corollaire 3.2 , lorsque $(k, \ell) \equiv\left(k_{0}, \ell_{0}\right)(\bmod 8)$, le terme principal obtenu est

$$
\begin{aligned}
\mathbf{1}_{m_{0}^{\prime} m_{12}^{\prime} n n^{\prime \prime} \ell^{\prime} \ell_{23}^{\prime}=1} \frac{\tilde{u}_{3}\left(m_{12}\right)}{k_{13} m_{0} m_{12}^{\prime} n^{\prime} n^{\prime \prime} k^{\prime}=1} & \frac{\mu^{2}\left(2 k_{13}^{\prime} \ell_{23}\right)}{\pi^{2+\alpha+\beta}} \frac{\varphi_{2}\left(k_{13}^{\prime} \ell_{23} m_{12}\right)}{m_{12}^{2} \ell_{23} k_{13}^{\prime}} \frac{\mu^{2}\left(n^{\prime \prime \prime}\right) \varphi_{2}\left(n^{\prime \prime \prime}\right)}{2^{\omega\left(n^{\prime \prime \prime} \ell_{23} k_{13}^{\prime} m_{12}\right)}}\left(\frac{n^{\prime \prime \prime}}{m_{12}}\right) \\
& \times \frac{P^{2}}{\log P}\left\{1+O\left(\frac{\left(\log 2 \ell_{23} k_{13}^{\prime} m_{12}\right)^{\frac{3}{2}}}{\log P}\right)\right\},
\end{aligned}
$$

avec

$$
\begin{aligned}
\tilde{u}_{3}\left(m_{12}\right)= & (-1)^{\vartheta\left(\ell_{0} \ell_{23} m_{12}\right) \vartheta\left(k_{13}^{\prime}\right)} u\left(k_{0}, \ell_{0} \ell_{23}, m_{12}\right)\left(\frac{\varepsilon_{2} 2^{\beta}}{k_{13}^{\prime}}\right)\left(\frac{2^{\gamma} \ell_{23} k_{13}^{\prime}}{m_{12}}\right) \\
& \times h_{2}\left(\varepsilon_{1} 2^{\alpha} k_{0} k_{13}^{\prime} m_{12}, \varepsilon_{2} 2^{\beta} \ell_{0} \ell_{23} m_{12}, 2^{\gamma} n^{\prime \prime \prime} k_{13}^{\prime} \ell_{23}\right) .
\end{aligned}
$$

Ensuite, nous sommons les $n^{\prime \prime \prime} \leqslant P /\left(2^{\gamma} k_{13}^{\prime} \ell_{23}\right)$ congrus à $n_{0}^{\prime \prime \prime}(\bmod 8)$ avec $n_{0}^{\prime \prime \prime}$ impair, premiers à $2 k_{13}^{\prime} \ell_{23}^{\prime}$ en appliquant le Lemme 3.1 avec $\nu=2$. Nous obtenons un terme principal

$$
\mathbf{1}_{m_{12}=1} \frac{\tilde{u}_{3}(1)}{\pi^{3}} \frac{\mu^{2}\left(2 k_{13}^{\prime} \ell_{23}\right)}{2^{4+\alpha+\beta+\gamma}} \frac{\varphi_{2}\left(k_{13}^{\prime} \ell_{23}\right) c_{2}\left(2 k_{13}^{\prime} \ell_{23}\right)}{2^{\omega\left(k_{13}^{\prime} \ell_{23}\right)}\left(k_{13}^{\prime} \ell_{23}\right)^{2}} \frac{P^{3}}{(\log P)^{\frac{3}{2}}}\left\{1+O\left(\frac{\left(\log 2 \ell_{23} k_{13}^{\prime}\right)^{\frac{3}{2}}}{\log P}\right)\right\} .
$$

Nous avons

$$
\begin{aligned}
\tilde{u}_{3}(1) & =(-1)^{\vartheta\left(\ell_{0} \ell_{23}\right) \vartheta\left(k_{13}^{\prime}\right)} u\left(k_{0}, \ell_{0} \ell_{23}, 1\right)\left(\frac{\varepsilon_{2} 2^{\beta}}{k_{13}^{\prime}}\right) h_{2}\left(\varepsilon_{1} 2^{\alpha} k_{0} k_{13}^{\prime}, \varepsilon_{2} 2^{\beta} \ell_{0} \ell_{23}, 2^{\gamma} n_{0}^{\prime \prime \prime} k_{13}^{\prime} \ell_{23}\right) \\
& =u\left(k_{0} k_{13}^{\prime}, \ell_{0} \ell_{23}, 1\right) h_{2}\left(\varepsilon_{1} 2^{\alpha} k_{0} k_{13}^{\prime}, \varepsilon_{2} 2^{\beta} \ell_{0} \ell_{23}, 2^{\gamma} n_{0}^{\prime \prime \prime} k_{13}^{\prime} \ell_{23}\right) .
\end{aligned}
$$


où nous avons utilisé la définition (4.4) de $u$. D'après les Lemmes 4.1 et 5.3 , nous avons

$$
\begin{aligned}
\sum_{(\alpha, \beta, \gamma) \in\{0,1\}^{3}} & \sum_{\substack{\varepsilon_{1}, \varepsilon_{2} \in\{ \pm 1\} \\
\left(\varepsilon_{1} k_{0} k_{13}^{\prime}, \varepsilon_{2} \ell_{2} \ell_{23}\right) \in E_{\alpha, \beta}(\bmod 8) \\
n_{0}^{\prime \prime \prime} \in(\mathbb{Z} / 8 \mathbb{Z})^{*}}} \frac{\tilde{u}_{3}(1)}{2^{4+\alpha+\beta+\gamma}} \\
= & \sum_{\substack{(\alpha, \beta, \gamma) \in\{0,1\}^{3} \\
\left(u_{0}, v_{0}\right) \in E_{\alpha, \beta}(\bmod 8) \\
w_{0} \in(\mathbb{Z} / 8 \mathbb{Z})^{*}}}(-1)^{\vartheta\left(\varepsilon_{1}\right) \vartheta\left(\varepsilon_{2}\right)} \frac{(-1)^{\vartheta\left(u_{0}\right) \vartheta\left(v_{0}\right)}}{2^{4+\alpha+\beta+\gamma}} h_{2}\left(2^{\alpha} u_{0}, 2^{\beta} v_{0}, 2^{\gamma} w_{0}\right) \\
= & \frac{\nu_{2}(-1)}{8}=\frac{17}{2} .
\end{aligned}
$$

Puis enfin une sommation sur $k_{13}$ et $\ell_{23}^{\prime}$ fournit une deuxième contribution à $N_{2}(P)$ égale à la moitié de (5.5).

Pour conclure, nous avons montré la formule

$$
N_{2}(P)=\frac{153}{8 \pi^{\frac{7}{2}}} \prod_{p} \frac{\left(1-\frac{1}{p}\right)^{\frac{1}{2}}}{\left(1+\frac{1}{p}\right)}\left(1+\frac{3}{2 p}+\frac{1}{p^{2}}\right) \frac{P^{3}}{(\log P)^{\frac{3}{2}}}\left\{1+O\left(\frac{1}{\log P}\right)\right\} .
$$

En combinant, dans (2.2), cette estimation avec (4.5), nous achevons la démonstration du Théorème 1.1.

\section{Références}

[1] M. Bhargava, A positive proportion of plane cubics fail the Hasse principle. En préparation, 2012.

[2] R. de la Bretèche et T.D. Browning, Density of Châtelet surfaces failing the Hasse principle. Soumis, 2012. (arXiv:1210.4010)

[3] J.-L. Colliot-Thélène, Groupe de Brauer non ramifié d'espaces homogènes de tores. Soumis, 2012. (arXiv: 1210.3644)

[4] J. Friedlander et H. Iwaniec, Ternary quadratic forms with rational zeros. J. Théor. Nombres Bordeaux 22 (2010), 97-113.

[5] J.-J. Sansuc, Groupe de Brauer et arithmétique des groupes algébriques linéaires sur un corps de nombres. J. reine angew. Math. 327 (1981), 12-80.

[6] J.-P. Serre, Cours d'arithmétique, Collection SUP : Le Mathématicien, 2, Presses Universitaires de France, Paris, (1970) 188 pp.

R. DE LA BRetÈche, Institut de Mathématiques de Jussieu, Université Denis Diderot, Case Postale 7012, F-75251 Paris CEDEX 13, France • E-mail : breteche@math.jussieu.fr

T.D. Browning, School of Mathematics, University of Bristol, Bristol, BS8 1TW, United Kingdom E-mail : t.d.browning@bristol.ac.uk 